\title{
The Analysis of Grade 8 Fractions Errors Displayed by Learners Due to Deficient Mastery of Prerequisite Concepts
}

\author{
Yeyisani Evans Makhubele ${ }^{1 \star(1)}$
}

\begin{abstract}
${ }^{1}$ University of Mpumalanga, Nelspruit, Mbombela, SOUTH AFRICA
\end{abstract}
*Corresponding Author: yeyisani@gmail.com

Citation: Makhubele, Y. E. (2021). The Analysis of Grade 8 Fractions Errors Displayed by Learners Due to Deficient Mastery of Prerequisite Concepts. International Electronic Journal of Mathematics Education, 16(3), em0645. https://doi.org/10.29333/iejme/11004

\begin{tabular}{|c|c|}
\hline ARTICLE INFO & ABSTRACT \\
\hline Received: 2 May. 2020 & This paper presents an analysis of fractions errors displayed by learners due to deficient mastery of prerequisite \\
\hline Accepted: 9 Jan. 2021 & $\begin{array}{l}\text { concepts. Fractions continue to pose a critical challenge for learners. Fractions can be a tricky concept for learners } \\
\text { although they often use the concept of sharing in their daily lives. } 30 \text { purposefully sampled learners participated } \\
\text { in this study. The research instrument consists of fractions test whose questions were selected from various } \\
\text { Annual National Assessment examination papers containing in addition, subtraction multiplication and division } \\
\text { of fractional operations. The different types of errors displayed by learners were then identified, coded and } \\
\text { categorized. The analysis showed that the main sources for errors were lack of understanding of the basic } \\
\text { concepts, learners' prior knowledge, misconceptions and misapplication of rules. This study recommends that } \\
\text { teachers should help their learners to develop fractions conceptual understanding. Learners need to be explicitly } \\
\text { taught that errors are opportunities for learning, and that they are springboard of inquiry. }\end{array}$ \\
\hline
\end{tabular}

Keywords: errors, fractions, proper fractions, conceptual understanding

\section{INTRODUCTION}

Fractions are notoriously difficult for students to understand and continue to pose a critical challenge for learners in South Africa (Baidoo, 2019). This paper presents an analysis of grade 8 fractions errors displayed by learners due to deficient mastery of prerequisite concepts. The poor performance of South African learners in internationally benchmarked literacy and numeracybased exams has been well documented in the literature (Jojo, 2019; Robertson \& Graven, 2020; Sibanda \& Graven, 2018; South African Institute of Race Relations, 2020; Spaull, 2019; van der Berg \& Gustafsson, 2019). One of the topic areas that learners display numerous errors in in Mathematics is fractions (Lemonidis \& Pilianidis, 2020; Reinholda et al., 2020). Research indicates that learners have difficulties with operations involving the addition, subtraction, division and multiplication fractions (Baidoo, 2019; Ubah \& Bansilal, 2018). One hypothesised reason for this difficulty is that students have memorised procedural rules and techniques for dealing with fractions without developing a corresponding conceptual understanding of fraction magnitudes, which makes many operational rules appear meaningless (Geller, Son, \& Stigler, 2017). Learners are displaying errors due to their deficient conceptual understanding of fractions. According to Wiest and Amankonah (2019), conceptual understanding involves seeing the connections between concepts and procedures, and being able to apply mathematical principles in a variety of contexts.

Fractions are one of the basic but poorly understood concepts in mathematics in elementary school curriculum. Students can understand simple issues whereas they have difficulty in learning more abstract concepts in fractions (Reinholda et al., 2020; Mohyuddin \& Khalil, 2016). Students do not try to understand the logic behind the fractional operations instead they memorize the rules, formulas, algorithms and terms (Wiest \& Amankonah, 2019). Students' development of more sophisticated concepts of fractions, beyond the part-whole concept, lays the groundwork for the later study of important mathematical topics, such as algebra, ratios, and proportions. In order to support such concepts, it is important to understand the underlying mental actions that undergird them so that teachers can design appropriate instructional opportunities. This study was therefore conducted to analyse grade 8 fractions errors displayed by learners due to deficient mastery of prerequisite concepts.

\section{CONTEXT AND BACKGROUND OF THE STUDY}

The study by Spaull (2019, p. 3) found that South Africa, together with other several of the world's 'Global South' countries participating in international and cross-national bench-marking assessments of literacy and numeracy have performed poorly 
relative to other countries. South Africa's TIMSS (Trends in International Mathematics and Science Study) assessment results indicated that ' $61 \%$ of the Grade 5 students could not add and subtract whole numbers, have no understanding of multiplication by one-digit numbers and cannot solve simple word problems, i.e., they cannot do basic mathematics' (Spaull 2019, p. 3).

Studies show that one of the reasons for this poor performance is Language of Learning and Teaching (Prediger, 2019; Prediger, Erath, \& Moser Opitz, 2019; Robertson \& Graven, 2020). Less than 5\% of South Africans are English first language speakers and the rest of the population is notably heterogeneous. This diversity within the group of second language learners complicates the matter of learning Mathematics via a second language even more. In South Africa's case, the indigenous African languages struggle to 'compete' with English in terms of their social, educational, political and economic traction within and beyond the country's borders (Robertson \& Graven, 2020). As 'minority' languages, they risk being overshadowed, even completely replaced, by the more prestigious language of power, social status and commerce (English) - which is also a widely spoken international language. Learning a new and difficult subject like mathematics through the medium of a second language gives learners a double task of mastering both mathematics content and language. This double task entails the acquisition of two conceptually difficult and different skills at once - one being related to language and the other to mathematics content.

The South African Language-in-Education Policy states that mother tongue should be the preferred medium of instruction in the Foundation Phase (Department of Basic Education, 2011). Primary tuition is therefore currently offered in the 11 South African official languages. The challenge faced in South African schools that offer African languages in the Foundation Phase, is the fact that from grade 4 onwards, education is only available through the medium of English. This results in a vast number of learners having to make a transition in grade 4 to English as medium of instruction. The philosophy behind this policy is that learners require a solid grounding in their home language in order to establish the skills necessary to engage with further learning. In Grade 4, learners switch to English as the language of learning and teaching. However, the majority of learners do not have enough exposure to English either at home or at school, to enable them to develop the English literacy skills necessary to cope with learning through the medium of English (Sibanda, 2017). Within this context, poor English language skills among learners have thus been identified as one of the key factors that impact negatively on mathematics results. The challenge therefore is that most learners in South African schools face a language barrier in the classroom. Ervin (2017) argues that any child who cannot use the language which he/she is most familiar with (usually the home language), is disadvantaged and unlikely to perform to the best of his/her ability.

Research indicates that learners perform poorly in subjects taught in an unfamiliar Language of Learning and Teaching, particularly in those that require decontextualised use of language such as Mathematics (Baidoo, 2019). One of the topic areas that learners display numerous errors in grade 8 Mathematics is fractions (Lemonidis \& Pilianidis, 2020). In their study, Lemonidis and Pilianidis (2020) observe that there is a great deal of agreement that learning rational number concepts remains a serious obstacle in the mathematical development of learners. Part of this difficulty may be due to the fact that the idea of a fraction is one of the earliest abstract ideas with which children have to cope since there is no natural context in which they automatically arise (Idris, 2018). Studies by Deringöl (2019) and Mulwa (2015) found that one of the learners' poor performance fractions calculations may be attributed mainly to affected learners' inadequate grasp of fractions concepts. The study made the following observations: some terms like quotient, numerator, and equivalent were given colloquial rather than mathematical interpretations. Some terms were confused with others possibly on the basis of the wrong assumption that they implied the same mathematical operation. Some terms were confused with their mathematical inverses.

\section{AIMS AND CONTRIBUTION TO KNOWLEDGE}

The main goal of this study was to analyse the grade 8 fractions errors displayed by learners due to deficient mastery of prerequisite concepts. This study contributes to teaching and learning fractions through the efforts of identifying and categorising errors. This research report has a potential of improving teachers' knowledge about the types of errors that learners are likely to generate during fraction instruction. It has a potential of improving their error handling skills.

\section{LITERATURE REVIEW}

\section{The Teaching of Fractions}

The study by Lemonidis and Pilianidis (2020) indicate that fractions are one of the most complex mathematical topics that learners encounter in their mathematics education. Researchers argue that this is because most teachers find it difficult to teach fractions (Lemonidis \& Pilianidis, 2020; Wilkins \& Norton, 2018). These researchers provided various reasons why fractions are difficult to understand and teach. The first reason is the failure to understand the logic behind fractional operations. Studies reveal that learners find it difficult to understand fractions because they do not try to understand the logic behind the fractional operations, instead they memorize the rules, formulas, algorithms and terms (Önal \& Yorulmaz, 2017; Wilkins \& Norton, 2018). This will result in the instrumental understanding of fractions (Purnomol et al., 2017).

The second reason is the multifaceted construct of fractions. Researchers and scholars agree that one of the predominant factors contributing to the complexities of teaching and learning fractions which lead to errors lies in the fact that fractions comprise a multifaceted construct (Norton, Wilkins, \& Xu, 2018). Fractions comprise a multifaceted notion encompassing five interrelated subconstructs, namely part-whole, ratio, operator, quotient, and measure (Strother et al., 2016). This result in 
fractions having many meanings. Errors will therefore occur when fractions are taught and generally reduced to only a meaning part-whole.

The third reason is textbook reliance. Most textbooks put more emphasis on procedures and steps to solve fractions problems (Loc, Tong, \& Chau, 2017). This cultivates the culture of rote memorization rather than having learners develop their own understanding of fraction concepts (Idris, 2018).

The fourth reason is the whole number bias. When learning fractions, sometimes learners use natural number properties to make inferences on rational numbers. Gabriel et al. (2013) called this a "whole numbers bias". This bias leads to difficulties conceptualizing whole numbers as decomposable units. Learners generally believe that the properties of whole numbers are the same for all numbers.

\section{Lack of Conceptual Understanding}

Studies indicate that fraction errors occurs as a result of lack of conceptual understanding that is critical for competence with fractions (Trivena, Ningsih, \& Jupri, 2017; Wilkins \& Norton, 2018). Such lack of conceptual understanding limits learners' ability to solve more advanced computational problems, including ratios, rates, and proportions, all of which are critical foundational skills for algebra. Reddy et al. (2016) identified language as a significant contributory factor to such poor performance, albeit not the only factor at work. These authors report that only 31\% of students in South Africa's TIMSS 2015 cohort 'always or almost always spoke the language of learning and teaching at home', achieving significantly better scores than those whose home language was different from the language used at school (Reddy et al., 2016, p. 8). These findings are consistent with those of Robertson and Graven (2020). In their study, Robertson and Graven (2020) noted that a prime cause for South African students' underachievement was that 'the pivotal role of language in education is neglected in curriculum and in teacher training programmes, resulting in limited language awareness, and consequently inadequate teaching methods that lead to language difficulties across all curriculum areas'. Edmonds-Wathen (2017) and Prediger et al. (2019) remind us that language is a major learning medium used for communicative and epistemic purposes in mathematics classrooms', making it imperative that language 'become a learning goal also, in mathematics classrooms.

\section{Challenging Concepts}

Idris (2018) states, a learner's ability to learn mathematics is directly related to his or her understanding of mathematical concepts and principles. Concepts are the basic building block of thinking, particularly higher-level thinking in mathematics. If children are to understand mathematics they have to learn the basic concepts and principles of mathematics. Concepts enable children to construct knowledge and communicate with others, and they are therefore important objects of thought. Fractions knowledge is based on several foundational concepts. Researchers agree on three critically important ideas and foundational concepts that should be developed for true fraction understanding, namely units and unitizing, partitioning and iterating, and equivalence (Barnett-Clarke et al., 2010; Mulwa, 2015).

\section{Unit and Unitizing}

According to Barnett-Clarke et al. (2010, p. 19) a unit fraction is the size of the counting piece. Determining the unit is key to interpretation and is important because it describes the size of some quantity with rational number. Schumacher and Malone (2017) argue that learners must be given tasks that help develop their idea of the counting unit and tasks must also give learners the opportunity to learn and apply the idea of a unit fraction. Unitizing is the renaming of the pieces or combining of units, for the purpose of counting in a new group (Lamon, 2012). Renaming is significant when finding equivalent fractions as well as with fraction computation of unlike denominators.

\section{Partitioning and Iterating}

Lamon (2012) describes partitioning as breaking or fracturing of a whole. It can also be described as dividing an object or objects into a number of disjoint and exhaustive parts. When a whole is partitioned, each of the parts is of equal area. Partitioning involves learners understanding that as the number of pieces increase in the whole or one; the smaller the pieces become. Iterating of fractions is the 'building up' of the unit piece. It is another way to make sense of fractions and improper fractions. When a unit is copied to create the one or whole, the unit has been iterated (Lamon, 2012).

\section{Equivalence}

Equivalence of fractions and decimals is the last foundational concept when developing understanding of rational number ideas (Lee \& Boyadzhiev, 2020). Lamon (2012) defines equal in part-whole fractions as the same in number, length, and area. In other words, many different fractions can name the same amount.

\section{METHODOLOGY AND DESIGN}

This is a qualitative case study located within an interpretive paradigm. The target population was grade eight learners. 30 learners were purposefully sampled and divided into three groups, according to their performance from previous assessments (ten high achievers, ten average achievers, ten low achievers). High achievers were the learners scoring between $70 \%$ and $100 \%$. Average achievers were those scoring between $45 \%$ and $69 \%$, and low achievers were those scoring less than $45 \%$. The instrument used in this study was a fractions test. The test questions were selected from various Annual National Assessment examination 
Table 1. Performance of learners in simplifying fractions

\begin{tabular}{|c|c|c|c|c|c|}
\hline Purpose & Question & $\begin{array}{c}\text { Number of } \\
\text { correct answers }\end{array}$ & $\begin{array}{l}\text { Percentage of } \\
\text { correct answers }\end{array}$ & $\begin{array}{c}\text { Number of } \\
\text { incorrect answers }\end{array}$ & $\begin{array}{c}\text { Percentage of } \\
\text { incorrect answers }\end{array}$ \\
\hline Add fractions with same denominator & $\frac{2}{8}+\frac{3}{8}+\frac{4}{8}$ & 25 & $83.33 \%$ & 5 & $16.67 \%$ \\
\hline Add fractions with different denominators & $\frac{1}{2}+\frac{5}{8}+\frac{3}{4}$ & 11 & $36.67 \%$ & 19 & $63.33 \%$ \\
\hline Subtract fractions with same denominators & $\frac{3}{4}-\frac{1}{4}-\frac{1}{4}$ & 21 & $67.74 \%$ & 9 & $32.26 \%$ \\
\hline Subtract fractions with different denominators & $\frac{1}{6}-\frac{2}{3}-\frac{1}{4}$ & 8 & $26.67 \%$ & 22 & $73.33 \%$ \\
\hline Division of fractions with same denominators & $\frac{3}{6} \div \frac{1}{6} \div \frac{5}{6}$ & 2 & $6.67 \%$ & 28 & $93.33 \%$ \\
\hline Division of fractions with different denominators & $\begin{array}{c}\frac{1}{5} \div \frac{3}{10} \div \\
\frac{4}{20}\end{array}$ & 4 & $13.33 \%$ & 26 & $86.67 \%$ \\
\hline Multiplication of fractions with same denominators & $\frac{1}{5} \times \frac{4}{5} \times \frac{3}{5}$ & 5 & $16.67 \%$ & 25 & $83.33 \%$ \\
\hline $\begin{array}{l}\text { Multiplication of fractions with different } \\
\text { denominators }\end{array}$ & $\frac{1}{2} \times \frac{3}{5} \times \frac{3}{9}$ & 7 & $23.33 \%$ & 23 & $76.67 \%$ \\
\hline
\end{tabular}

Table 2. Categories of errors

\begin{tabular}{|c|c|}
\hline Code & Description of errors with examples \\
\hline C1 & Non - systematic errors. These are slips, lapses or unintended mistakes. E.g., $\frac{1}{2}-\frac{1}{4}=\frac{1}{4}-\frac{1}{4}=\frac{2}{4}=\frac{1}{2}$. \\
\hline $\mathbf{C 2}$ & $\begin{array}{l}\text { Ignorance of rule restriction or symbolism. These refer to extension of previously available strategies in new situations where they do } \\
\qquad \text { not apply/ misapplication of rule, e.g., } \frac{1}{2}+\frac{2}{3}=\frac{3}{5}\end{array}$ \\
\hline C3 & $\begin{array}{l}\text { Incomplete application of rule. Correct application of the first step of a rule, but fail to proceed, e.g., } \frac{1}{4}+\frac{1}{2}=1+\frac{1}{4}=\frac{2}{4}=\frac{1}{2} .(\text { this happens } \\
\qquad \text { when learners are still developing the rules of the structure they are learning) }\end{array}$ \\
\hline C4 & Conceptual error. Lack of conceptual understanding. Does not understand the properties, e.g., $\frac{5}{9}-\frac{2}{8}=\frac{45}{72}-\frac{24}{72}=\frac{21}{72}=\frac{7}{21}$. \\
\hline
\end{tabular}

papers set by the Department of Basic Education in South Africa. The obtained data were analysed via the content analysis technique. To ensure the content validity the test was piloted and validated before being used in the actual study.

\section{FINDINGS}

We coded the sampled learners L1 to L30. In the data presentation below, L represents learner and will always be followed by a number.

\section{Summary of the Analysis of the Sampled Learners' Responses when Simplifying Proper Fractions}

Table 1 displays the number and percentage of correct and incorrect answers in the different operations of fractions. The analysis of the majority of learners' scripts shows that learners have challenges with fractions.

The results as displayed in Table 1 show that many learners display errors through incorrect answers. They had challenges in the additions of fractions with different denominators (63.33\% incorrect answers), subtractions of fractions with different denominators (73.33\% incorrect answers), division of fractions with same denominators (93.33\% incorrect answers), division of fractions with different denominators (86.67\% incorrect answers), multiplication of fractions with same denominators (83.33\% incorrect answers), and multiplication of fractions with different denominators (76.67\% incorrect answers)

We then coded the errors displayed in learners' work from C1 to C4, as shown in Table 2. Code 1 represents non - systematic errors, code 2 ignorance of rule restriction or symbolism, code 3 incomplete applications of rules, and code 4 conceptual errors.

We then analysed the learners' errors in terms of these codes. The results are displayed in Table 3. 
Table 3. Different types of errors displayed by learners

\begin{tabular}{|c|c|c|c|}
\hline Kind of operation & Type of error & Number of learners & Percentage (\%) \\
\hline \multirow{5}{*}{ Addition } & $\mathrm{C} 1$ & 1 & 3.3 \\
\hline & $\mathrm{C} 2$ & 4 & 13.3 \\
\hline & $\mathrm{C} 3$ & 11 & 36.7 \\
\hline & $\mathrm{C} 4$ & 3 & 10 \\
\hline & None & 11 & 36.7 \\
\hline \multirow{5}{*}{ Subtraction } & $\mathrm{C} 1$ & 3 & 10 \\
\hline & $\mathrm{C} 2$ & 6 & 20 \\
\hline & $\mathrm{C} 3$ & 9 & 30 \\
\hline & $\mathrm{C} 4$ & 5 & 16.75 \\
\hline & None & 7 & 23.3 \\
\hline \multirow{5}{*}{ Multiplication } & $\mathrm{C} 1$ & - & 0 \\
\hline & $\mathrm{C} 2$ & 20 & 66.7 \\
\hline & $\mathrm{C} 3$ & 6 & 20 \\
\hline & $\mathrm{C} 4$ & 2 & 6.7 \\
\hline & None & 2 & 6.7 \\
\hline \multirow{5}{*}{ Division } & $\mathrm{C} 1$ & 1 & 3.3 \\
\hline & $\mathrm{C} 2$ & 16 & 53.3 \\
\hline & $\mathrm{C} 3$ & 9 & 30 \\
\hline & $\mathrm{C} 4$ & - & 0 \\
\hline & None & 4 & 13.3 \\
\hline
\end{tabular}

Table 4. Types of errors displayed by the different sampled groups

\begin{tabular}{|c|c|c|c|c|}
\hline & $\begin{array}{c}\text { Non- systematic errors } \\
\text { (C1) }\end{array}$ & $\begin{array}{l}\text { Ignorance of rule } \\
\text { restriction errors (C2) }\end{array}$ & $\begin{array}{c}\text { Incomplete application of } \\
\text { rule error }(\mathrm{C} 3)\end{array}$ & $\begin{array}{c}\text { Conceptual errors } \\
\text { (C4) }\end{array}$ \\
\hline High achievers & 2 & 9 & 10 & 3 \\
\hline Average achievers & 2 & 14 & 10 & 3 \\
\hline Low achievers & - & 20 & 14 & 2 \\
\hline Total & 4 & 43 & 34 & 8 \\
\hline
\end{tabular}

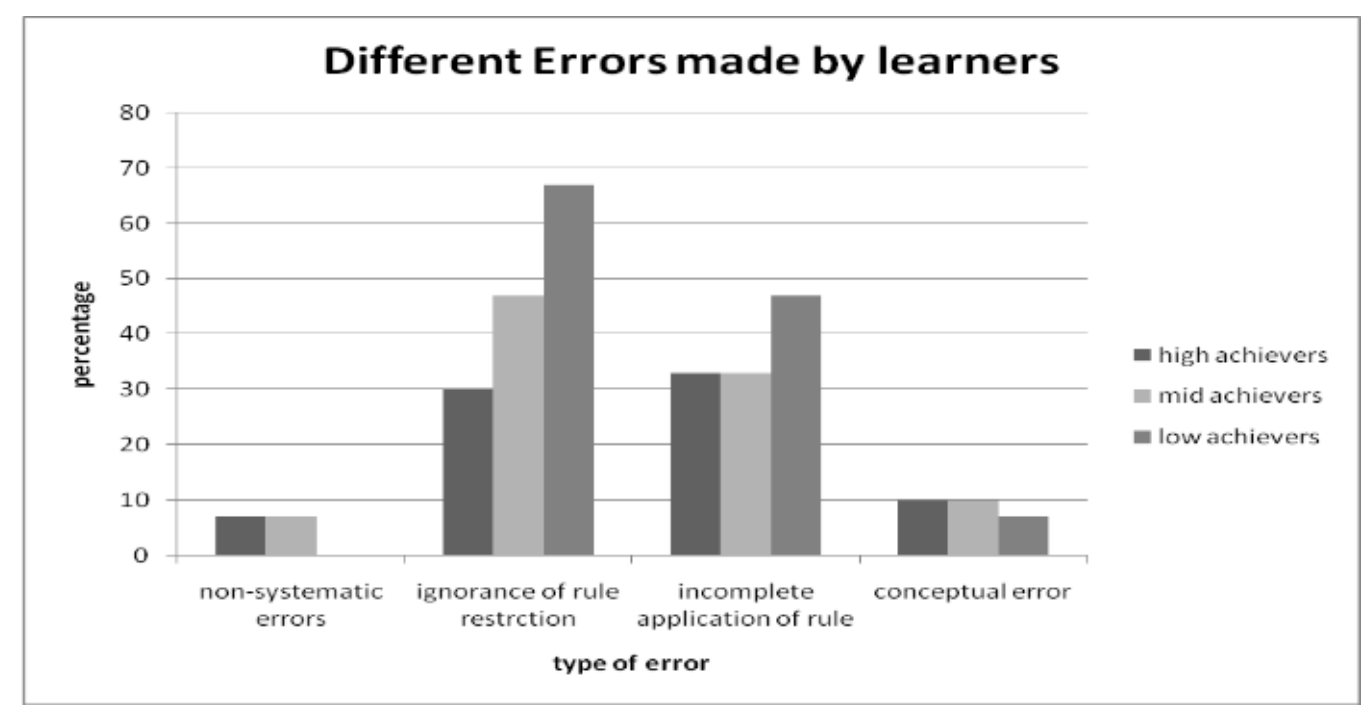

Figure 1. Different errors committed by learners of different abilities

Table 3 shows that learners committed more C 3 errors in addition, subtraction and division. C2 errors were mostly committed in multiplication and division. $\mathrm{C} 1$ errors were the least committed. This is displayed in Table 4 which shows how many learners from the different categories committed errors. C2 and C3 errors were also prevalent in the high and achievers' scripts.

Figure 1 shows a graphical representation of the different types of errors displayed by learners.

\section{Analysis of Learners' Responses to Addition of Proper Fractions}

Learners were asked to add three pairs of fractions with the same denominators and one with different denominators (refer to Table 5 for questions and the percentage results). 
Table 5. High achievers' responses to addition of fractions

\begin{tabular}{ccccc}
\hline Purpose & Questions & $\begin{array}{c}\text { Number of } \\
\text { correct answers }\end{array}$ & $\begin{array}{c}\text { Percentage of } \\
\text { correct answers }\end{array}$ & $\begin{array}{c}\text { Number of } \\
\text { incorrect answers }\end{array}$ \\
\hline $\begin{array}{c}\text { Pdncorrect answers } \\
\text { denominator }\end{array}$ & (1a) $\frac{2}{8}+\frac{3}{8}+\frac{4}{8}$ & 25 & 83.33 & 5 \\
\hline $\begin{array}{c}\text { Add fractions with different } \\
\text { denominators }\end{array}$ & (1b) $\frac{1}{2}+\frac{5}{8}+\frac{3}{4}$ & 11 & 36.67 & 19 \\
\hline
\end{tabular}

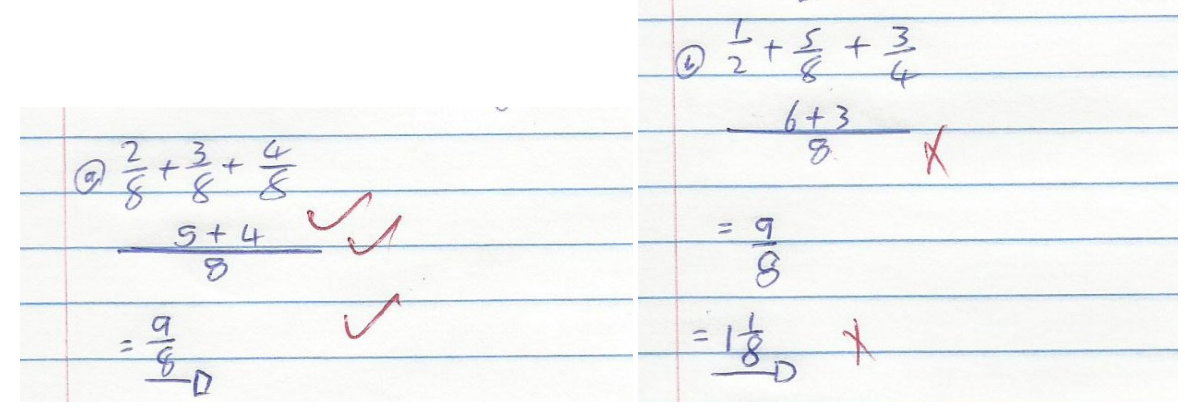

Figure 2. High achiever's response to addition of proper fractions

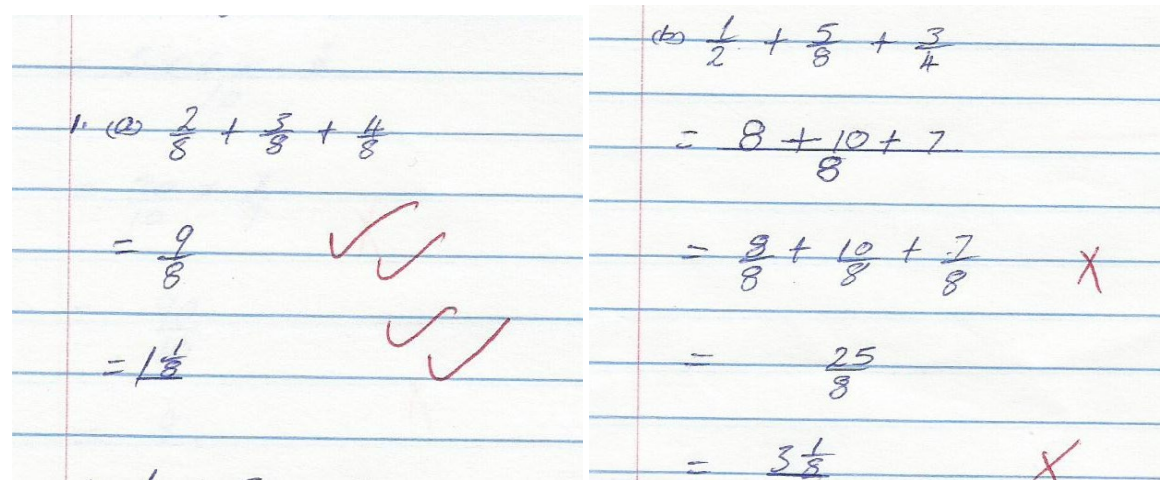

Figure 3. Average achiever's response to addition of proper fractions

Table 5 clearly shows that learners display most errors when adding fractions with different denominators. In question 1 (a) $83.33 \%$ of the learners responded correctly to the addition of fractions with the same denominators while $16.67 \%$ responded incorrectly. In question 1 (b), $36.67 \%$ of the learners responded correctly to the addition of fractions with different denominators while 63, 33\% responded incorrectly. Figure 2 shows one of the responses by one the high achievers. L2 managed to simplify fractions with same denominators in question 1 (a) but failed to simplify fractions with different denominators in question 1 (b). The learner used the wrong strategy to get incorrect answer. This learner got the common denominator of 2,4 , and 8 which is 8 , but added the numerators as they are. This indicates that learners have difficulties in finding common denominators more especially in more than two pairs of fractions.

While L18, one of the average achievers managed to get the correct answer in question 1 (a), in question 1 (b) the learner made some computational error, when adding fractions with different denominators (see Figure 3). This learner failed to make numerators to be accord with denominators. He divided 8 by 2 which is 4 and multiplied the answer by 2 , the denominator not numerator. In the second fraction L18 multiplied 5 by 2 instead of 5 by 1 . In the third fraction this learner added the numerator and denominator, $3+4=7$.

Majority of the low achievers are the ones who responded incorrectly in this question. Most of them added the numerators and denominators together. This confirms the results of earlier research by Siegler and Lortie-Forgues (2015) that in addition of fractions learners tend use 'add tops, add bottoms'. Figure 4 shows the response by one of the low achievers.

The analysis clearly shows that that most learners are good in adding fractions with common denominators but have challenges in adding fractions with different denominators. 


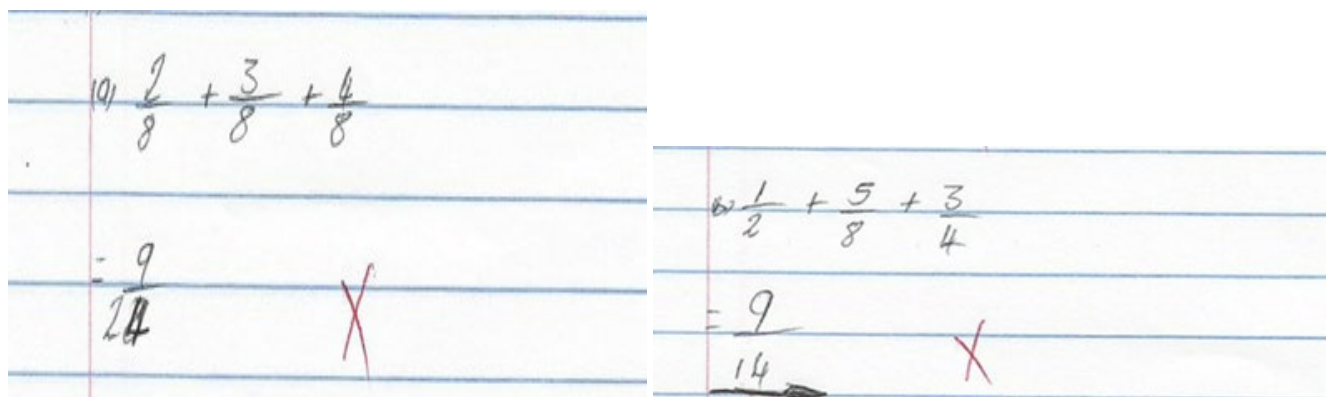

Figure 4. Low achiever's response to addition of proper fractions

Table 6. Performance of learners in subtraction of fractions

\begin{tabular}{ccccc}
\hline Purpose & Questions & $\begin{array}{c}\text { Number of } \\
\text { correct answers }\end{array}$ & $\begin{array}{c}\text { Percentage of } \\
\text { correct answers }\end{array}$ & $\begin{array}{c}\text { Number of } \\
\text { incorrect answers }\end{array}$ \\
\hline $\begin{array}{c}\text { Subtract fractions with same } \\
\text { denominators }\end{array}$ & (2a) $\frac{3}{4}-\frac{1}{4}-\frac{1}{4}$ & 21 & 67.74 & 9 \\
\hline $\begin{array}{c}\text { Subtract fractions with different } \\
\text { denominators }\end{array}$ & (2b) $\frac{1}{6}-\frac{2}{3}-\frac{1}{4}$ & 8 & 26.67 & 22 \\
\hline
\end{tabular}

$\begin{aligned} & \frac{3}{4}-\frac{1}{4} \frac{1}{4} \\ = & \frac{3-1}{4} \\ = & \frac{2}{4} \div 2 \\ = & \frac{1}{2}\end{aligned}$

Figure 5. Average achiever's response (L15) to subtraction of proper fractions

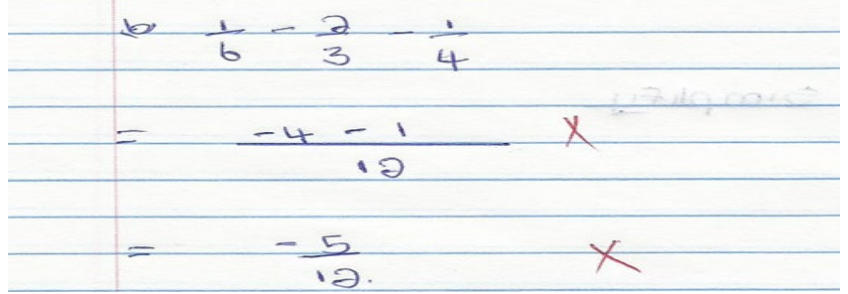

Figure 6. Average achiever's response to subtraction of proper fractions

\section{Analysis of Learners' Responses to Subtraction of Proper Fractions}

Learners were asked to subtract three pairs of fractions with same denominators and one with different denominators (refer to Table 6 for questions and the percentage results). In question 2(a) $67.74 \%$ of the learners responded correctly to subtraction of fractions with same denominators while $32.26 \%$ responded incorrectly. In question 2(b), $26.67 \%$ of the learners responded correctly to subtraction of fractions with different denominators while $73.33 \%$ responded incorrectly.

Most high achievers performed extremely well in this question involving the subtractions of fractions (see Figure 5).

Figure 5 shows one of the average achievers' responses to subtraction of fractions with different denominators. In question 2(a), L15 got the correct lowest common denominator which is 4, but failed to subtract the numerators.

In question 2(b), L15 also got the correct lowest common denominator of 3, 4, and 6 which is 12, but failed to make numerators to be accord with their denominators (see Figure 6). The learner added numerators to get - 4 but added another -1 to get $\frac{-5}{12}$. This learner made a C2 error, namely the incomplete application of a rule as indicated in Figure 6.

The majority of learners in this group applied incorrect strategies to get incorrect answers. The reasons are the lack of conceptual understanding of common denominators and the lack of background knowledge of equivalent fractions. Another reason could be that teachers used more procedural (instrumental) approach when teaching fractions so learners tend to memorise without deep understanding. Figure 7 shows the response by L18, who added numerators instead of subtracting in question 2(a). In question 2(b) the learner chose the larger denominator, 6 instead of using the Lowest common denominator 12. 


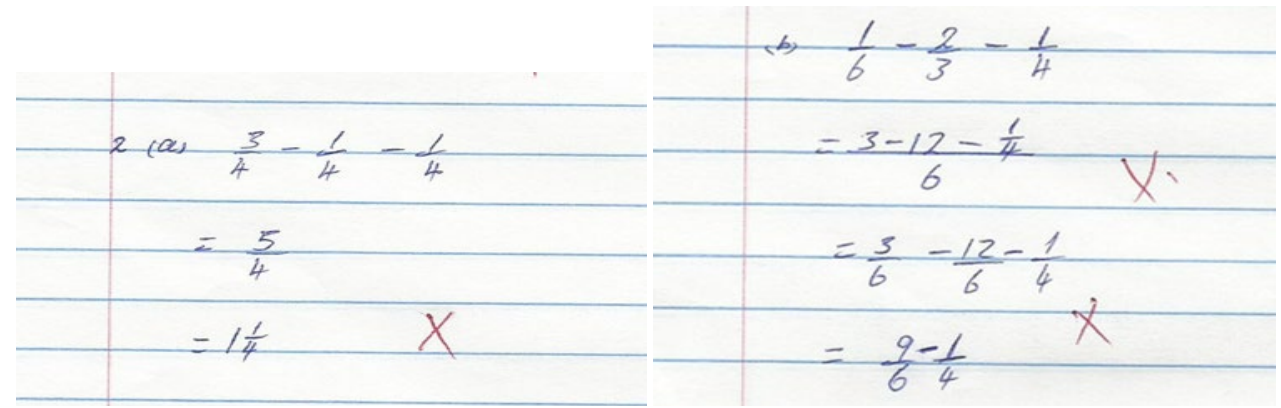

Figure 7. Average achiever's response to subtraction of proper fractions

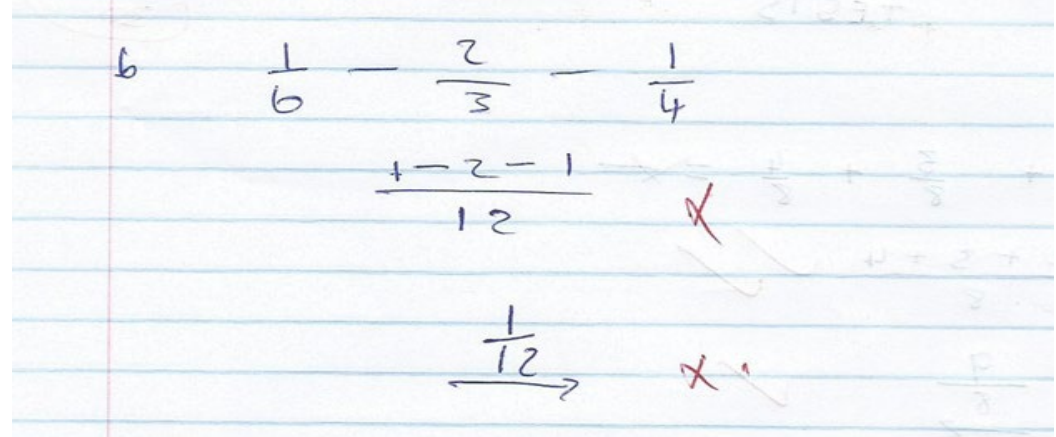

Figure 8. Low achiever's response to subtraction of proper fractions

Table 7. Performance of learners in division of fractions

\begin{tabular}{cccccc}
\hline Purpose & Questions & $\begin{array}{c}\text { Number of } \\
\text { correct answers }\end{array}$ & $\begin{array}{c}\text { Percentage of } \\
\text { correct answers }\end{array}$ & $\begin{array}{c}\text { Number of } \\
\text { incorrect answers }\end{array}$ & $\begin{array}{c}\text { Percentage of } \\
\text { incorrect answers }\end{array}$ \\
\hline $\begin{array}{c}\text { Division of fractions with same } \\
\text { denominators }\end{array}$ & $(3 \mathrm{a}) \frac{3}{6} \div \frac{1}{6} \div \frac{5}{6}$ & 2 & 6,67 & 28 & 93,33 \\
\hline $\begin{array}{c}\text { Division of fractions with different } \\
\text { denominators }\end{array}$ & $(3 \mathrm{~b}) \frac{1}{5} \div \frac{3}{10} \div \frac{4}{20}$ & 4 & 13,33 & 26 & 86,67 \\
\hline
\end{tabular}

The majority of the low achievers did not manage to get correct answers. One of the underachievers, L28 made C2 error, namely the incomplete application of a rule. She got the correct common denominator of 3, 4, and 6 but subtracted the numerators without changing them to correspond with their denominators. Learners tend to make mistakes when working with negative numbers as shown beneath in Figure 8.

Like in the additions of fractions, our analysis show that most learners are good in subtracting fractions with common denominators but have challenges in subtracting fractions with different denominators.

\section{ANALYSIS OF LEARNERS' RESPONSES TO DIVISION OF PROPER FRACTIONS}

The majority of the learners found division to be more challenging (refer to Table 7 for questions and the percentage results). In question 3(a) $6.67 \%$ of the learners responded correctly to division of fractions with same denominators while $93.33 \%$ responded incorrectly. In question 3(b), $11.33 \%$ of the learners responded correctly to division of fractions with different denominators while $86.67 \%$ responded incorrectly. Most learners have no relational understanding about algorithm invert and multiply. This means they do not know why they are inverting the second fraction and multiply. Table 7 shows how learners performed in division of fractions.

Figure 9 shows one of the responses by one of the high achievers. In question 3(a) L8 did not invert the second fraction. He inverted the third fraction. The learner cross cancelled the numerator of the first fraction (3) and the denominator of the second fraction (6) to get denominator of 2. The learner then proceeded simplifying the second and third fraction without using his answer which was 2. The learner displayed a misconception of inverting the last pair of fraction when given three pairs of fractions. He therefore made $\mathrm{C} 3$ error, namely the incomplete application of rule. 


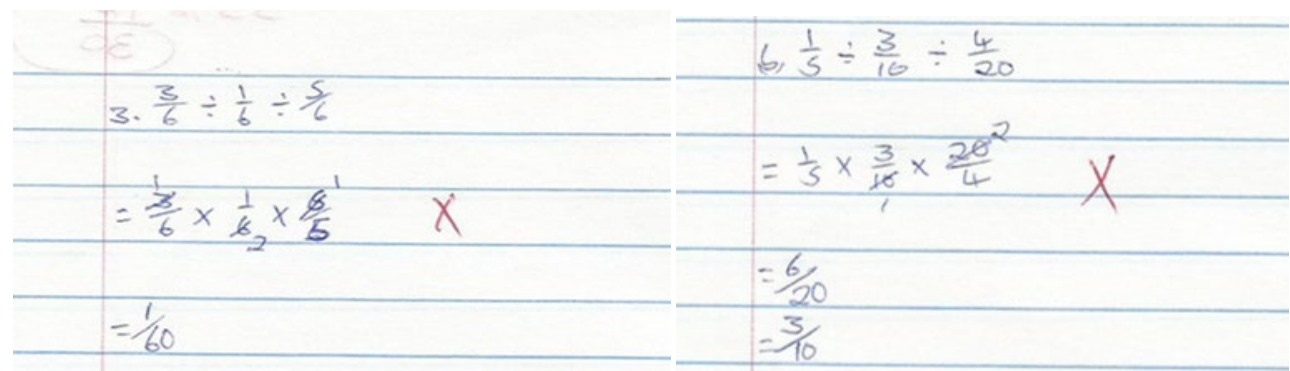

Figure 9. High achiever's response to division of proper fractions

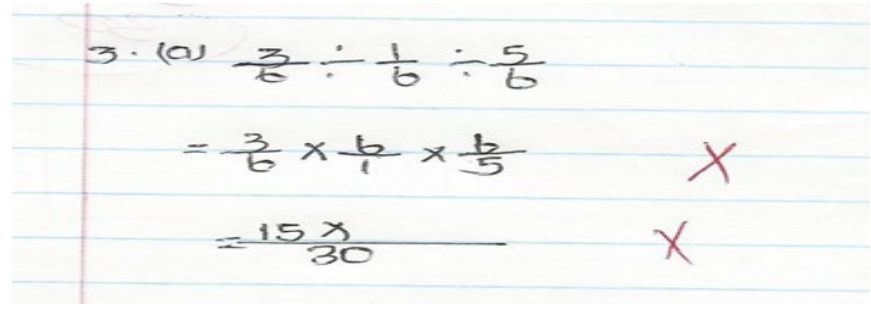

Figure 10. Average achiever's response to division of proper fractions

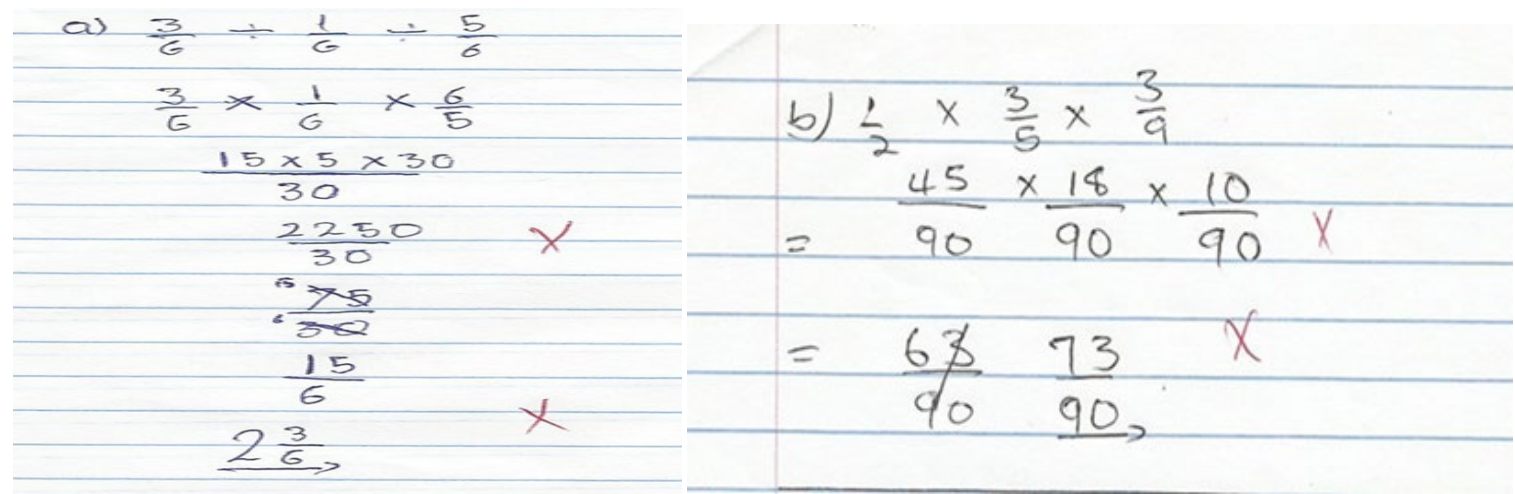

Figure 11. High achiever's response to multiplication of proper fractions

In question 3(b) L8 made the same error of inverting the third fraction and cross cancelled. He multiplied the numerators to get the product of numerators and denominators.

Most mid achievers displayed errors in this question. L14 from this group, for example correctly inverted the second and third fraction, but made $\mathrm{C} 2$ and $\mathrm{C} 3$ errors. This learner knew the first step of finding the reciprocals of the last two fractions but failed to proceed. He found the lowest common denominator of 5 and 6 which is 30 although common denominators are only restricted to addition and subtraction of fractions. Multiplication of fractions is simply getting the product of numerators and denominators. Figure 10 is example of response by L14.

Figure 10 displays an error displayed by one of the learners from the average achievers which is similar to L14 who inverted the third fraction and used the wrong strategy of lowest common denominators.

Low achievers did not perform well in the division of proper fractions. They committed many errors, see for example, Figure 11. L30, for example used the incorrect strategy of finding common denominators to division of fractions with same denominators in question 3(a). This learner displayed misconception of finding common denominators to any pairs of fractions. In question 3(b) the learner chose the larger denominator and divided the numerators of the three pairs of fractions as shown in Figure 11.

The learner made a C3 error, namely ignorance of rule restrictions. Common denominators are restricted to addition and subtraction of fractions.

\section{Analysis of Learners' Responses to Multiplication of Proper Fractions}

Like in division, learners also did not perform well in the multiplication of proper fractions (refer to Table $\mathbf{8}$ for questions and the percentage results). In question 4 (a) $16.67 \%$ of the learners responded correctly to multiplication of fractions with same denominators while $83.33 \%$ responded incorrectly. In question $4(\mathrm{~b}), 23.33 \%$ of the learners responded correctly to multiplication of fractions with different denominators while $76.67 \%$ responded incorrectly. Although rules of multiplication are easy to work with, the majority of the learners found it to be challenging. Multiplication involves multiplying the numerators together and denominators together. 
Table 8. Performance of learners in multiplication of fractions

\begin{tabular}{ccccc}
\hline Purpose & Questions & $\begin{array}{c}\text { Number of } \\
\text { correct answers }\end{array}$ & $\begin{array}{c}\text { Percentage of } \\
\text { correct answers }\end{array}$ & $\begin{array}{c}\text { Number of } \\
\text { incorrect answers incorrect answers }\end{array}$ \\
\hline $\begin{array}{c}\text { Multiplication of fractions with same } \\
\text { denominators }\end{array}$ & (4a) $\frac{1}{5} \times \frac{4}{5} \times \frac{3}{5}$ & 5 & 16,67 & 25 \\
\hline $\begin{array}{c}\text { Multiplication of fractions with different } \\
\text { denominators }\end{array}$ & (4b) $\frac{1}{2} \times \frac{3}{5} \times \frac{3}{9}$ & 7 & 23,33 & 23 \\
\hline
\end{tabular}

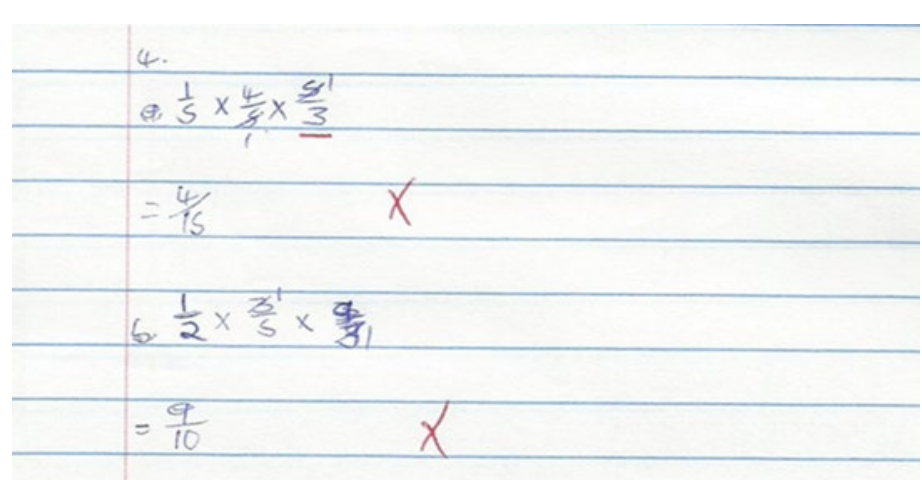

Figure 12. High achiever's response to multiplication of proper fractions

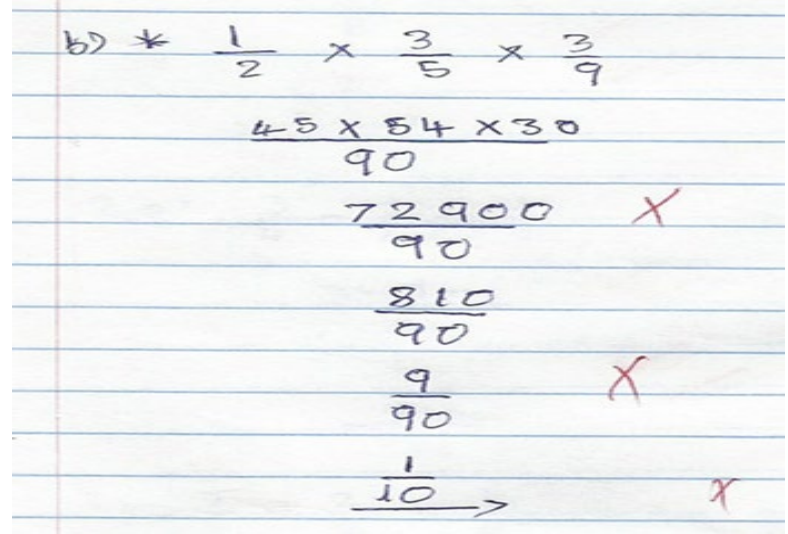

Figure 13. Average achiever's response to multiplication of proper fractions

High achievers performed extremely well in this question. Some learners from this group, for example L6, displayed some errors (see Figure 12). This learner used the correct strategy to find the product of the denominators. He applied the knowledge of common denominators to multiplication and changing the corresponding numerator of the first fraction only. He then added the numerators together, $45+18+10=73$.

L8, another high achiever, displayed an error of flipping the third fraction to $\frac{5}{3}$. The learner cross cancelled the denominator of the second fraction with the numerator of the third fraction and multiplied the numerators and denominators to get $\frac{4}{15}$ (refer to Figure 13).

The same error was committed by average achievers. They found the inverse of the third fraction and cross cancelled.

Low achievers also experienced some challenges in this question. Figure $\mathbf{1 4}$ shows an example of one of the learners from the low achievers who applied knowledge of common denominators to multiplication of fractions. The learner used an incorrect strategy to get the correct answer. The learner used the common denominator of 90 and changed their corresponding numerators and got the product of 72900 . She divided the product by 90 to get a proper fraction of $\frac{9}{90}$, and reduced it to $\frac{1}{10}$.

In question 4(a) another learner from this group used the rule of addition and subtraction to multiplication (see Figure 14). This learner used the denominator of 5 and multiplied the numerators to get the product of $\frac{12}{5}$. In question 4 (b) the same learner found the common denominator of 2, 5 and 9 and multiplied the numerators and got the correct answer. 


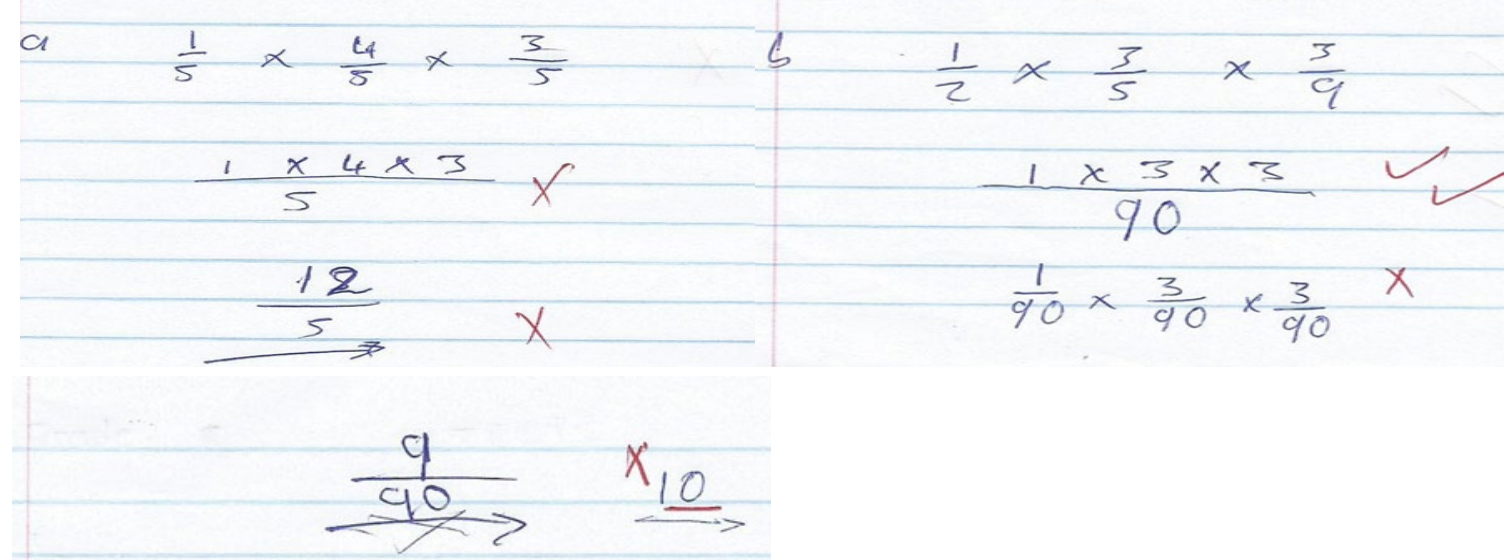

Figure 14. Low achiever's response to multiplication of proper fractions

\section{Findings of Learners' Responses on Addition of Proper Fractions}

* Adding numerators to any pairs of given fractions and choosing the larger denominator. Learners have the tendency of adding numerators without changing them accord to their denominator, e.g., $\frac{1}{2}+\frac{5}{8}+\frac{3}{4}=\frac{1+5+3}{8}=\frac{9}{8}=1 \frac{1}{8}$. Most learners failed to find common denominators when adding fractions with unlike denominators, so they usually used the easy way of choosing the larger denominator.

* Adding numerators and denominators. The majority of the low achievers displayed this error, for example $\frac{1}{2}+\frac{3}{8}=\frac{4}{10}$. The findings are similar to earlier research by Brown and Quinn (2006) where learners used whole number schema to fractions.

* Lack of conceptual understanding of fractions. Some learners made some computation errors in addition of proper fraction, for example: $\frac{1}{2}+\frac{3}{8}=\frac{1}{3} \times \frac{2}{8}=\frac{6+8}{24}=\frac{14}{24}$. This is applying the flipping rule of the second denominator which is applied in the division of fractions. This show shows a lack of relational understanding of fractions. The reason could be when fractions are taught rules are overemphasized, rather the understanding, and rules are easily forgotten.

* Flipping the second fraction to addition of proper fractions. This error was displayed by a majority of the low achievers as a result of memorising rules, for example: $\frac{1}{4}+\frac{1}{2}=\frac{1}{4} \times \frac{2}{1}=\frac{8}{1}=8$. Learners do not know when and why do we find the reciprocal of the first fraction. When they add fractions they usually flipped the second fraction even though this are only restricted to division of fractions. This is a C3 error, ignorance of rule restrictions.

\section{Findings on Learners' Responses to Subtraction of Proper Fractions}

- Failing to subtract proper fractions with like denominators. This error was displayed by both low achievers and average achievers for example: $\frac{3}{4}-\frac{1}{4}-\frac{1}{4}=\frac{3-1}{4}=\frac{2}{4}=\frac{1}{2}$. It seems they were mostly grounded on application of rules or procedural understanding.

* Changing denominators of fractions without changing the corresponding numerators. This was displayed by most of the underachievers who showed a lack of relational understanding of equivalent fractions, for example: $\frac{1}{6}-\frac{2}{3}-\frac{1}{4}=\frac{-4-1}{12}=\frac{-5}{12}$. Most of them managed to get the lowest common denominators but failed to change their corresponding numerators. This is a typical C2 error.

* Failing to get common denominators. Most of the underachievers displayed the error of choosing the larger denominator when they have to subtract or add three pairs of fractions for example: $\frac{3}{5}-\frac{5}{6}=\frac{3-5}{6}=\frac{-2}{6}$. This is an indication that learners lack conceptual knowledge of solving fractions with different denominators. The reason could be that when teaching fractions, teachers stressed the use of rules or procedures which causes some of the learners to apply rules where they do not apply.

* Subtracting numerators and denominators. Learners' responses showed that some learners are confused by fractions and whole numbers. Learners of subtract numerators together and denominators e.g., $\frac{3}{4}-\frac{1}{4}=\frac{2}{0}$.

\section{Findings of Learners' Responses to Multiplication of Proper Fractions}

* Use of common denominators in multiplication of proper fractions. These learners were applying the addition rule of fractions to multiplication, for example: $\frac{2}{6} \times \frac{3}{6}=\frac{6}{6}=1$. Some learners will get the common denominator and also change the corresponding numerators and multiply the find the product of the numerators and product of denominators. $\frac{1}{2} \times \frac{3}{5} \times \frac{3}{9}=\frac{45 \times 54 \times 30}{90}=\frac{72900}{90}=\frac{810}{90}=\frac{9}{90}=\frac{1}{10}$. These learners display lack of conceptual knowledge of multiplication of fractions and improper fractions. 
* Inverting fractions when simplifying the final answer. The most common error made by most of the underachievers when multiplying fractions is to multiply the numerators and denominators, followed by flipping the fraction product, for example: $\frac{2}{6} \times \frac{3}{6}=\frac{6}{36}=6$. This shows that learners have confusion with proper and improper fractions, but to some learners this is as a result of not revisiting their work (non- systematic errors).

* Finding the reciprocal of the second or third fraction in multiplication. Some learners flip the second or third fraction and cross cancelled, for example: $\frac{1}{5} \times \frac{4}{5} \times \frac{3}{5}=\frac{1}{5} \times \frac{4}{5} \times \frac{5}{3}=\frac{4}{15}$. Learners flipped $\frac{3}{5}$ to $\frac{5}{3}$, then cross cancelled the denominator of the second fraction and numerator of the third fraction and multiplied the numerators and denominators to get $\frac{4}{15}$. Learners made the error becuse they confused multiplication with division.

* Finding common denominators and add. Some learners in this research used the common denominator to simplify fractions which are multiplied. This shows a confusion of applying the addition rule of fractions to multiplication of fractions, e.g., $\frac{7}{8} \times \frac{2}{4}=\frac{14+32}{8}=\frac{46}{8}$.

\section{Findings of Learners' Responses on Division of Proper Fractions}

- Applying knowledge of like and unlike denominators to division of fractions. Learners tend to apply knowledge of like and unlike to division of fractions, e.g. $\frac{3}{5} \div \frac{1}{5}=\frac{3}{5}$. Learners determined the denominator of 5 without inverting the second fraction.

* Changing the division sign to multiplication without flipping the second fraction. Learners sometimes do not remember the algorithm invert and multiply, they only remember the word multiply. After changing the division sign, they proceed to multiply numerators and denominators together, e.g: $\frac{3}{5} \div \frac{7}{8}=\frac{3}{5} \times \frac{7}{8}=\frac{14}{56}=4$. Learners are not aware that diving by a fraction is the same as multiplying by its inverse. This is an indication that learners only memorise procedure without understanding.

* Cross cancelling without finding the inverse of the second fraction. Learners usually multiply numerators together and denominators together without finding the reciprocal of the second faction as in multiplication, e.g.: $\frac{2}{7} \div \frac{7}{8}=\frac{1}{4}$. The reason could be teachers teach algorithm which causes poor conceptual understanding. This confirms the results by Siemon (2004), were he asserted that learners misuse the invert and multiply procedure to fractions.

\section{DISCUSSION}

The responses given by learners demonstrated considerable deficiency in their conceptual understanding of fractions. The findings reveal that most learners display errors as a result a low level of conceptual understanding. Learners have developed misconceptions and have very poor understanding of concepts, procedures, and appropriate application. Conceptual errors occur when learners have misconceptions or misunderstanding about the concepts related to the problem, such as the concept of how to add two fractions (Wiest \& Amankonah, 2019). These conceptual errors are produced when learners have incorrect knowledge of the underlying structure of mathematics, that is, they lack understanding of the relationships and interconnections of ideas that explain and give meaning to fractions procedures. When solving fractional equations, it became evident in learners' errors that they lack conceptual understanding which results in lack of procedural skills such as the steps involved in solving fractional equations and the rules governing those steps (algorithms). They lack relational understanding. These findings are consistent with those of (Baidoo, 2019). Fractions errors made by learners included incorrect understanding of a mathematical concepts, application of wrong procedures and generalising rules merely after seeing it working in a few particular instances. This study therefore reveal that learners were too dependent on algorithms and on the memorisation of formulas and rules.

\section{CONCLUSION}

This study focused on grade 8 fractions errors displayed by learners due to deficient mastery of prerequisite concepts. Misunderstanding of fractions concepts will always hinder students' learning of operations with rational expressions, exponents, and other algebraic operations. Most common errors were not only due to the inability to compute with fractions but also a lack of understanding of basic fraction concepts. Understanding learners' conceptual deficiencies is an essential step towards effective teaching of fractions. It is therefore important for teachers to help their learners develop conceptual understanding of fractions. The aim of learning mathematics is building learners' knowledge of mathematical concepts. Fractions teaching should therefore also focus on the understanding of fractions concepts. Conceptual understanding enables learners to solve fractions problems in various forms and novel settings. Learners with high levels of conceptual knowledge are capable of solving problems that they have never come across before. Hence, a reformation in teaching is needed to boost conceptual understanding among learners in order to minimise the use of algorithms and memorization. 


\section{IMPLICATIONS FOR FRACTIONS TEACHING}

How errors are dealt with in a mathematics classroom is important as it can either support or deny learner access to mathematical knowledge. Errors play a central role in the mathematics classroom as they are a reflection of the manner in which learners reason and they illuminate the processes through which learners attempt to construct their own knowledge. It is for this reason that we call on teachers to change their pedagogical instructional practices when teaching fractions. When teaching fractions, teachers need to be on the lookout for students' common misconceptions that lead to errors in computation. Instead of emphasizing the memorisation of algorithms in fraction lessons, they should help learners develop a deep understanding of fractions concepts and identify connections between these concepts. The problem is that traditional pedagogical fractions practices are deeply ingrained and focus on algorithms without first ensuring conceptual understanding. Algorithms are not inherently bad; the key to using them to help rather than hinder understanding lies in the sequence of events that occur in learning fractions concepts. Fractions instruction must first ensure that learners' conceptual understanding is deeply embedded. When learners have truly mastered a concept, they should be able to show all the detailed steps in a process, explain why those steps occur, and connect the process to related concepts. Once learners reach this level of understanding, a teacher can then expose them to more efficient ways to express or perform those same processes. Relying only on algorithms and procedures and focusing on shortcuts results in teaching efficiency, not mathematics.

We recommend a conceptual oriented instruction of fractions that enables learners to achieve a level of computation competence they would not have achieved had they been in a procedurally oriented math class. Conceptually oriented instruction enhances learners' ability to understand, and through these understandings computational competence is achieved. This will provide opportunities for learners to explore arithmetic concepts in many different ways. This will assist then learners to acquire the vocabulary and correct phraseology of fractions. The traditional drill and practice curriculum and instruction provides learners with only one way to solve a computational problem - using a memorised algorithm. Teachers can encourage conceptual discourse by allowing learners to speak informally about fractions - exploring, explaining, and arguing their interpretations and ideas. This kind of exploratory talk is important for learners to develop ideas and concepts in a comfortable environment. It is also important for enabling teachers to listen to learners' ideas and conceptions so that these can be worked with and built upon. According to Robertson and Graven (2020), it is in this environment of informal exploratory talk that learners begin to acquire conceptual discourse.

We also call on teachers to avoid naked numbers when teaching fractions. The phrase naked numbers refers to the prevalent use of fractions in isolation, without any descriptors, units, or context. Most teachers continue to employ traditional instruction methods that use naked numbers, especially in the form of drill and practice geared to learner memorisation of facts or procedures. As a result, learners can easily lose sight of the meaning of fractions, resulting in a diminished view of fractions. Without any context, learners alike forget the meaning of fractions and the key idea that they represent something.

Teachers should be aware of the benefits and limitations of correcting, probing and embracing errors. Using their professional knowledge, teachers should decide when and why it is appropriate to correct, to probe and to embrace errors in light of their knowledge of the content and their learners. Teachers should embrace errors as springboards for learning.

Funding: No funding source is reported for this study.

Declaration of interest: No potential conflict of interest was reported by the author.

\section{REFERENCES}

Baidoo, J. (2019). Dealing with grade 10 learners' misconceptions and errors when simplifying algebraic fractions. Journal of Emerging Trends in Educational Research and Policy Studies (JETERAPS), 10(1), 47-55.

Barnett-Clarke, C., Fisher, W., Marks, R., \& Ross, S. (2010). Rational numbers: The big ideas and essential understandings. In R. Charles \& R. Zbiek (Eds.), Developing essential understanding of rational numbers for teaching mathematics in grades 3-5 (pp. 10-56). The National Council of Teachers of Mathematics, Inc.

Brown, G., \& Quinn, R. J. (2006). Algebra students' difficulty with fractions: An error analysis. Australian Mathematics Teacher, 62(4), 28-40.

Denzin, N. K., \& Lincoln, Y. S. (2000). Introduction: The discipline and practice of qualitative research. In N. K. Denzin \& Y. S. Lincoln (Eds.), Handbook of qualitative research (pp. 1-28). Sage Publications.

Department of Basic Education. Republic of South Africa. (2011). Curriculum and assessment policy statement grades 1-3: English first additional language. Government Printing Works.

Deringöl, Y. (2019). Misconceptions of primary school students about the subject of fractions. International Journal of Evaluation and Research in Education (IJERE), 8(1), 29-38. https://doi.org/10.11591/ijere.v8i1.16290

Edmonds-Wathen, C. (2017). Responding to the mathematics curriculum with language and culture. Journal of Mathematics and Culture, 11(3), 36-63.

Ervin, H. K. (2017). Fraction multiplication and division models: A practitioner reference paper. International Journal of Research in Education and Science (IJRES), 3(1), 258-279.

Gabriel, F., Coché, F., Szucs, D., Carette, V., Rey, B., \& Content, A. (2013). A componential view of children's difficulties in learning fractions. Frontiers in Psychology, 4, 715. https://doi.org/10.3389/fpsyg.2013.00715 
Geller, E. H., Son, J. Y., \& Stigler, J. W. (2017). Conceptual explanations and understanding fraction comparisons. Learning and Instruction, 52, 122-129. https://doi.org/10.1016/j.learninstruc.2017.05.006

Idris, N. (2018). Error patterns in addition and subtraction of fractions among form two students. Journal of Mathematics Education, $4(2), 35-54$.

Jojo, Z. (2019). Mathematics education system in South Africa, education systems around the World. Intech Open. https://doi.org/10.5772/intechopen.85325

Lamon, S. (2012). Teaching fractions and ratios for understanding: Essential content knowledge and instructional strategies. Taylor \& Francis Group. https://doi.org/10.4324/9780203803165

Lee, H.-J., \& Boyadzhiev, I. (2020). Underprepared college students' understanding of and misconceptions with fractions. International Electronic Journal of Mathematics Education, 15(3), em0583. https://doi.org/10.29333/iejme/7835

Lemonidis, C., \& Pilianidis, N. (2020). The 8th grade students' competencies in alternating different symbolic representations of rational numbers. International Electronic Journal of MathematicsEducation, 15(3), em587. https://doi.org/10.29333/iejme/7865

Loc, N. P., Tong, D. H., \& Chau, P. T. (2017). Identifying the concept "fraction" of primary school students: The investigation in Vietnam. Educational Research and Review, 12(8), 531-539. https://doi.org/10.5897/ERR2017.3220

Mohyuddin, R. G., \& Khalil, U. (2016). Misconceptions of students in learning mathematics at primary level. Bulletin of Education and Research, 38(1), 133-162.

Mulwa, E. C. (2015). Difficulties encountered by students in the learning and usage of mathematical terminology: A critical literature review. Journal of Education and Practice, 6(13), 27-38.

Norton, A., Wilkins, J. L. M., \& Xu, C. Z. (2018). A progression of fraction schemes common to Chinese and U.S. students. Journal for Research in Mathematics Education, 49(2), 210-226. https://doi.org/10.5951/jresematheduc.49.2.0210

Önal, H., \& Yorulmaz, A. (2017). The errors made by primary school fourth graders on fractions. Journal of Research in Education and Society, 4(1), 98-113, 2017.

Prediger, S. (2019). Investigating and promoting teachers' expertise for language-responsive mathematics teaching. Mathematics Education Research Journal, 31, 367-392. https://doi.org/10.1007/s13394-019-00258-1

Prediger, S., Erath, K., \& Moser Opitz, E. (2019). Language challenges for students with mathematics difficulties: An overview on research results and instructional approaches. In A. Fritz, V. Haase, \& P. Räsänen (Eds.), International handbook of math learning difficulties: From the laboratory to the classroom (pp. 437-455). Springer. https://doi.org/10.1007/978-3-319-971483_27

Purnomol, Y. W, Widowati, C, Aziz, T. A., \& Pramudiani, P. (2017). Fractions division knowledge of elementary school student: The case of Lala. The 4th International Conference on Research, Implementation, and Education of Mathematics and Science (4th ICRIEMS), pp. 1-8. https://doi.org/10.1063/1.4995148

Reddy, V., Isdale, K., Juan, A., Visser, M., Winnaar, L., \& Arends, F. (2016). TIMSS 2015: Highlights of mathematics achievement of grade 5 South African learners. Department of Basic Education/ Human Sciences Research Council.

Reinhold, F., Hoch, S., Werner, B., Richter-Gebert, J., \& Reissa, K. (2020). Learning fractions with and without educational technology: What matters for high-achieving and low-achieving students? Learning and Instruction, 65, 101264. https://doi.org/10.1016/j.learninstruc.2019.101264

Robertson, S.-A., \& Graven, M. (2020). Language as an including or excluding factor in mathematics teaching and learning. Mathematics Education Research Journal, 32, 77-101. https://doi.org/10.1007/s13394-019-00302-0

Schumacher, R. F., \& Malone, A. S. (2017). Error patterns with fraction calculations at fourth grade as a function of students' mathematics achievement status. Elementary School Journal, 118(1), 105-127. https://doi.org/10.1086/692914

Sibanda, L. (2017). Grade 4 learners' linguistic difficulties in solving mathematical assessments. African Journal of Research in Mathematics, Science and Technology Education, 21(1), 86-96. https://doi.org/10.1080/18117295.2017.1291476

Sibanda, L., \& Graven, M. (2018). Can mathematics assessments be considered valid if learners fail to access what is asked of them? South African Journal of Childhood Education, 8(1), a505. https://doi.org/10.4102/sajce.v8i1.505

Siegler, R. S., \& Lortie-Forgues, H. (2015). Conceptual knowledge of fraction arithmetic. Journal of Educational Psychology, 107(3), 909-918. https://doi.org/10.1037/edu0000025

Siemon, D. (2004). Partitioning-The missing link in building fraction knowledge and confidence. RMIT University.

South African Institute of Race Relations. (2020). Freeing Education The future of the class of 2020: How to build a bright education future. The South African Institute of Race Relations.

Spaull, N. (2019). Priorities for education reform in South Africa (input document for Treasury's economic colloquium 19 January 2019). https://nicspaull.files.wordpress.com/2019/01/v2-spaull-priorities-foreduc-reform-treasury-19-jan-2019.Pdf

Strother, S., Brendefur, J. L., Thiede, K., \& Appleton, S. (2016). Five key ideas to teach fractions and decimals with understanding. Advances in Social Sciences Research Journal, 3(2), 132-137. https://doi.org/10.14738/assrj.32.1832

Trivena, V, Ningsih, A. R., \& Jupri, A. (2017). Misconception on addition and subtraction of fraction at primary school students in fifth-grade. International Conference on Mathematics and Science Education (ICMSCE), Journal of Physics: Conference Series 895 (2017) 012139. https://doi.org/10.1088/1742-6596/895/1/012139 
Ubah, I. J. A., \& Bansilal, S. (2018). Pre-service primary Mathematics teachers' understanding of fractions: An action-processobject-schema perspective. South African Journal of Childhood Education, 8(2), a539. https://doi.org/10.4102/sajce.v8i2.539

Van der Berg, S., \& Gustafsson, M. (2019). Educational outcomes in post-apartheid South Africa: Signs of progress despite great inequality. In N. Spaull \& J. Jansen (Eds.), South African Schooling: The Enigma of inequality. Springer. https://doi.org/10.1007/978-3-030-18811-5_2

Wiest, L. R., \& Amankonah, F. O. (2019). Conceptual versus procedural approaches to ordering fractions. European Journal of Science and Mathematics Education, 7(1), 61-72. https://doi.org/10.30935/scimath/9534

Wilkins, J. L. M., \& Norton, A. (2018). Learning progression toward a measurement concept of fractions. International Journal of STEM Education, 5, 27. https://doi.org/10.1186/s40594-018-0119-2 